\title{
Identification of critical amino acid residues in the plague biofilm Hms proteins
}

Correspondence
Robert D. Perry
rperry@pop.uky.edu

Received 15 June 2006

Revised 28 July 2006

Accepted 9 August 2006

\author{
Stanislav Forman, Alexander G. Bobrov, Olga Kirillina, Susannah K. Craig, \\ Jennifer Abney, Jacqueline D. Fetherston and Robert D. Perry
}

Department of Microbiology, Immunology, and Molecular Genetics, University of Kentucky, Lexington, Kentucky 40536-0084, USA

\begin{abstract}
Yersinia pestis biofilm formation causes massive adsorption of haemin or Congo red in vitro as well as colonization and eventual blockage of the flea proventriculus in vivo. This blockage allows effective transmission of plague from some fleas, like the oriental rat flea, to mammals. Four Hms proteins, $\mathrm{HmsH}, \mathrm{HmsF}, \mathrm{HmsR}$ and $\mathrm{HmsS}$, are essential for biofilm formation, with $\mathrm{HmsT}$ and $\mathrm{HmsP}$ acting as positive and negative regulators, respectively. $\mathrm{HmsH}$ has a $\beta$-barrel structure with a large periplasmic domain while HmsF possesses polysaccharide deacetylase and COG1649 domains. $\mathrm{HmsR}$ is a putative glycosyltransferase while $\mathrm{HmsS}$ has no recognized domains. In this study, specific amino acids within conserved domains or within regions of high similarity in $\mathrm{HmsH}$, $\mathrm{HmsF}, \mathrm{HmsR}$ and $\mathrm{HmsS}$ proteins were selected for site-directed mutagenesis. Some but not all of the substitutions in $\mathrm{HmsS}$ and within the periplasmic domain of $\mathrm{HmsH}$ were critical for protein function. Substitutions within the glycosyltransferase domain of $\mathrm{HmsR}$ and the deacetylase domain of $\mathrm{HmsF}$ abolished biofilm formation in $Y$. pestis. Surprisingly, substitution of highly conserved residues within COG1649 did not affect HmsF function.
\end{abstract}

\section{INTRODUCTION}

An intimate association of bacterial cells with a surface and formation of multicellular aggregates is commonly referred to as a biofilm. The organization of these bacterial communities is vastly diverse and dependent upon the production of extracellular substances constituting unique biofilm matrices. Such bacterial architectures are environmentally beneficial and can contribute to dissemination and virulence of an organism (Branda et al., 2005; Davey \& O’Toole, 2000).

The Yersinia pestis $\mathrm{Hms}^{+}$phenotype is a manifestation of biofilm formation causing massive adsorption of haemin or Congo red (CR) during growth at $26-34{ }^{\circ} \mathrm{C}$ but not at $37^{\circ} \mathrm{C}$. This is required for colonization and eventual blockage of the flea proventriculus - allowing efficient transmission of plague from some fleas, like the oriental rat flea, to mammals (Bacot \& Martin, 1914; Bacot, 1915; Hinnebusch et al., 1996; Lillard et al., 1997, 1999; Pendrak \& Perry, 1991, 1993; Perry et al., 1990, 1993, 2004; Perry \& Fetherston, 1997; Pollitzer, 1954). Biofilm formation is not involved in mammalian virulence - an in-frame deletion in $h m s R$ did not affect the $\mathrm{LD}_{50}$ in a bubonic plague model (Lillard et al., 1999). Recent studies have demonstrated Hms-dependent biofilm

Abbreviations: CR, Congo red; CV, crystal violet; EPS, exopolysaccharide. A table of primers is available with the online version of this paper. formation in vitro as well as in fleas and nematodes (Darby et al., 2002; Jarrett et al., 2004; Jones et al., 1999; Kirillina et al., 2004). CR binds basic and neutral polysaccharides while wheat germ agglutinin binds to $\mathrm{N}$-acetylglucosamine, suggesting that the Hms-dependent biofilm contains an exopolysaccharide (EPS) component. In addition, dispersin, an enzyme from Actinobacillus actinomycetemcomitans which hydrolyses $\beta$-1,6- $N$-acetyl-D-glucosamine, prevents plague biofilm formation but does not disrupt a preformed biofilm (Itoh et al., 2005; Spiers et al., 2002; Tan \& Darby, 2004; Wood, 1980).

The $102 \mathrm{~kb}$ pgm locus in $Y$. pestis contains the highpathogenicity island (Ybt) and the hmsHFRS operon encoding four of the six $h m s$ genes required for biofilm formation and its regulation. Escherichia coli K-12 pgaABCD (formerly $y c d S R Q P$ ) encode proteins with similarities (ranging from 34 to $83 \%$ ) to $\mathrm{Hms}$ proteins and are required for the synthesis of an EPS containing poly- $\beta-1,6-N$-acetylD-glucosamine (Blattner et al., 1997; Deng et al., 2002; Fetherston et al., 1992; Jones et al., 1999; Lillard et al., 1997; Wang et al., 2004). $\mathrm{HmsH}$ and $\mathrm{HmsF}$ are outer-membrane proteins while HmsS, HmsR, and HmsT are located in the inner membrane. While $\mathrm{HmsH}$ is predicted to be a $\beta$-barrel protein with a large periplasmic domain, its function and that of HmsS are unknown. HmsR, a putative glycosyltransferase, and $\mathrm{HmsF}$, with a polysaccharide deacetylase domain, are likely involved in the synthesis and modification of the EPS component of the biofilm. HmsF also has a 
conserved but uncharacterized domain, COG1649, that is present in other HmsF-like proteins in Gram-negative bacteria (Kirillina et al., 2004; Pendrak \& Perry, 1991; Perry et al., 2004). Proteins with this domain are assigned to the TIM barrel glycosyl hydrolase superfamily by Pfam (Bateman et al., 2004). Recently, gmhA, a gene encoding phosphoheptose isomerase, has been identified as important for biofilm formation (Darby et al., 2005).

Temperature regulation of plague biofilm formation does not involve transcriptional regulation or mRNA stability. Rather, this is achieved by proteolytic degradation of HmsR, $\mathrm{HmsH}$ and $\mathrm{HmsT}$ at $37^{\circ} \mathrm{C}$. $\mathrm{HmsT}$, a putative diguanylate cyclase, may be the key to this temperature regulation. It is a positive regulator of biofilm formation and is involved in the synthesis of cyclic-di-GMP (c-di-GMP). HmsP, a negative regulator of biofilm formation, is an EAL phosphodiesterase and likely degrades c-di-GMP. Like other glycosyltransferases involved in EPS or cellulose productions, the enzymic activity of HmsR may depend upon c-di-GMP (Bobrov et al., 2005; Hare \& McDonough, 1999; Jones et al., 1999; Kirillina et al., 2004; Perry et al., 2004; Simm et al., 2005).

Previously, we used site-directed amino acid substitutions to identify several critical residues of $\mathrm{HmsT}$ and $\mathrm{HmsP}$ (Kirillina et al., 2004). In this study, specific amino acids within conserved domains or within regions of high similarity in $\mathrm{HmsH}, \mathrm{HmsF}, \mathrm{HmsR}$ and $\mathrm{HmsS}$ were selected for site-directed mutagenesis. Substitutions within the glycosyltransferase domain of $\mathrm{HmsR}$ and the deacetylase domain of $\mathrm{HmsF}$ abolished biofilm formation in Y. pestis while substitutions in $\mathrm{HmsS}$ and $\mathrm{HmsH}$ had diverse effects. Surprisingly, substitution of highly conserved residues within COG1649 did not affect HmsF function.

\section{METHODS}

Bacterial strains and cultivation. All bacterial strains used in this study are described in Table 1. E. coli cells were grown on Luria broth agar (LBA). Where appropriate, ampicillin (Ap; $100 \mu \mathrm{g} \mathrm{ml}^{-1}$ ), kanamycin $\left(\mathrm{Km} ; 50 \mu \mathrm{g} \mathrm{ml}^{-1}\right)$, streptomycin $\left(\mathrm{Sm} ; 50 \mu \mathrm{g} \mathrm{ml}^{-1}\right)$ or chloramphenicol $\left(\mathrm{Cm} ; 20 \mu \mathrm{g} \mathrm{ml}^{-1}\right)$ were added to cultures. Strains with arabinose-inducible expression were grown in the presence of $0 \cdot 2 \%(\mathrm{w} / \mathrm{v})$ arabinose. $Y$. pestis cells were streaked onto Congo red (CR) plates from buffered glycerol stocks stored at $-80^{\circ} \mathrm{C}$ and incubated at $26^{\circ} \mathrm{C}$ for $48 \mathrm{~h}$. For most experiments, individual colonies were inoculated onto Tryptose Blood Agar Base (TBA; Difco) slants and incubated at $26^{\circ} \mathrm{C}$ for $24-48 \mathrm{~h}$. Cells were washed off TBA slants with Heart Infusion Broth (HIB; Difco) and grown overnight with aeration in HIB at the appropriate temperature. Cultures were transferred to fresh medium the next morning. For some experiments, Y. pestis cells were grown in TMH medium (Straley \& Bowmer, 1986). Cell growth was monitored on a Spectronic Genesys 5 spectrophotometer at $620 \mathrm{~nm}$. For Western blot analyses, isolated colonies from TBA plates were spread onto CR plates and allowed to form a lawn $(48 \mathrm{~h})$. Cells were scraped off the plate and suspended in sample buffer.

Crystal violet (CV) staining. Cells attached to borosilicate glass test tubes were detected with CV staining essentially as described by O’Toole et al. (1999). Briefly, cells were grown overnight on TBA slants and used to inoculate $\mathrm{TMH}$ to an $\mathrm{OD}_{620}$ of $0 \cdot 1$. Cultures were grown for $16-18 \mathrm{~h}$ in a shaking water bath at $26^{\circ} \mathrm{C}$ and then exposed to $0 \cdot 01 \% \mathrm{CV}$ for $15-20 \mathrm{~min}$. Cells attached to the culture tube were washed three times with distilled water and the CV staining the cells was solubilized with a mixture of $80 \%$ ethanol $/ 20 \%$ acetone. The amount of dye solubilized, representing the mass of attached bacterial cells, was measured at $570 \mathrm{~nm}$ on a Spectronic Genesys5 spectrophotometer.

CR-binding assay. The original CR-binding assay (Hartzell et al., 1999) was modified as previously described (Kirillina et al., 2004). Briefly, $Y$. pestis cells were grown overnight $(\approx 16 \mathrm{~h})$ in $\mathrm{HIB}$ at $26^{\circ} \mathrm{C}$. Cells were pelleted and resuspended in HIB/CR medium $[1 \%$ (w/v) HIB containing $0.2 \%$ galactose and $30 \mu \mathrm{g} \mathrm{CR} \mathrm{ml}{ }^{-1}$ ] such that all cultures had an equivalent wet weight of cells per $\mu$ l of medium. An $\approx 20 \mathrm{mg}$ wet cell weight aliquot of each culture was incubated for $3 \mathrm{~h}$ on a rocking platform at room temperature. The amount of $\mathrm{CR}$ bound by the cells was determined by measuring the absorbance of cell-free supernatants at $500 \mathrm{~nm}$ with a Spectronic Genesys $5 \mathrm{spec}-$ trophotometer and subtracting this value from the reading obtained with uninoculated HIB/CR medium.

Plasmids and recombinant DNA techniques. All the plasmids used in this study are listed in Table 1. Plasmids were purified from overnight cultures by alkaline lysis (Birnboim \& Doly, 1979) or with a Qiaprep spin Miniprep kit (Qiagen) and further purified when necessary by polyethylene glycol precipitation (Humphreys et al., 1975). A standard $\mathrm{CaCl}_{2}$ protocol was followed to introduce plasmids into E. coli (Sambrook \& Russell, 2001). Y. pestis cells were transformed by electroporation as previously described (Fetherston et al., 1995). All plasmid DNA and PCR products used for construction of chromosomal deletion mutants or cloned amino acid substitution mutants were sequenced by Elim Biopharmaceuticals to ensure that the correct mutation had been introduced and no secondary mutations had occurred. In a few instances, aberrant amino acid substitution clones were identified and these were also characterized.

Construction of hmsH, hmsR, hmsF and hmsS mutants. Suicide vector pKNG101 (Kaniga et al., 1991) was used to generate in-frame deletions in $h m s H$ and $h m s R$. Fragments spanning the upstream and downstream regions of $h m s H$ were obtained by NsiI/ XmnI (1147 bp) and SwaI/BamHI (1149 bp) digestions of pNPM22 and cloned into the PstI-BamHI sites of pBluescript SK. The resulting construct, pBSSK $\Delta \mathrm{hmsH}$, and pKNG101 were both digested with SalI and $X b a \mathrm{I}$ followed by ligation of the resulting $2 \cdot 4 \mathrm{~kb}$ fragment from pBSSK $\Delta \mathrm{hmsH}$ into pKNG101, yielding pKNG $\Delta \mathrm{hmsH}$ (Table 1). For the hmsR deletion, $1 \cdot 1 \mathrm{~kb}$ Sall-NcoI and $1 \cdot 1 \mathrm{~kb}$ BsrBI-BamHI fragments of the upstream and downstream region of $h m s R$ in pHMS1.2 were cloned into the SalI-BamHI sites of pWSK29, yielding pWSK29 $\Delta \mathrm{hmsR}$. This plasmid was digested with SalI $/ \mathrm{XbaI}$ and the resulting $2 \cdot 2 \mathrm{~kb}$ fragment was cloned into the same sites in pKNG101, yielding pKNG $\Delta$ hmsR (Table 1).

PCR and DNA sequencing (Elim Biopharmaceuticals) confirmed that the correct DNA sequences were present in pKNG $\Delta \mathrm{hmsH}$ and pKNG $\Delta$ hmsR prior to electroporation into $Y$. pestis KIM6+. As previously described (Bearden \& Perry, 1999), transformants were selected on TBA plates containing $50 \mu \mathrm{g} \mathrm{Sm} \mathrm{ml}^{-1}$ and grown overnight in HIB without antibiotics to select for sucrose-resistant isolates that had completed the allelic exchange. Strains in which the wild-type gene was replaced by the deleted version were identified by PCR. One isolate of each mutation was selected and named KIM6-2115 (in-frame $\Delta h m s H 2115$ ) and KIM6-2118 (non-polar $\Delta h m s R 2118$ ). These deletion mutations remove 569 of 822 and 327 of 457 aa in $\mathrm{HmsH}$ and HmsR, respectively.

Red-mediated recombination (Datsenko \& Wanner, 2000) was employed to construct non-polar deletions in $h m s F$ and $h m s S$ in $Y$. 
Table 1. Bacterial strains and plasmids

\begin{tabular}{|c|c|c|}
\hline Strain or plasmid & Relevant characteristics $\dagger$ & Source or reference \\
\hline \multicolumn{3}{|l|}{ Y. pestis ${ }^{\star}$} \\
\hline $\mathrm{KIM} 6+$ & $\mathrm{Pgm}^{+}\left(\mathrm{Hms}^{+}\right) \mathrm{Pla}^{+}$ & Fetherston et al. (1992) \\
\hline KIM6-2115 & $\mathrm{Hms}^{-}$(in-frame $\Delta h m s \mathrm{H} 2115$ ) $\mathrm{Pla}^{+}$ & This study \\
\hline KIM6-2116 & $\mathrm{Hms}^{-}$(non-polar $\Delta h m s F 2116$ ) $\mathrm{Pla}^{+}$ & This study \\
\hline KIM6-2118 & $\mathrm{Hms}^{-}$(in-frame $\Delta h m s R 2118$ ) $\mathrm{Pla}^{+}$ & This study \\
\hline \multicolumn{3}{|l|}{ E. coli } \\
\hline DH5 $\alpha$ & Cloning strain & Ausubel et al. (1987) \\
\hline $\mathrm{DH} 5 \alpha(\lambda$ pir $)$ & Strain for maintenance of R6K origin suicide plasmid & S. C. Straley, University of Kentucky \\
\hline \multicolumn{3}{|l|}{ Plasmids } \\
\hline pBluescript SK & $2 \cdot 9 \mathrm{~kb}, \mathrm{Ap}^{\mathrm{r}}$, high-copy-number cloning vector & Stratagene \\
\hline $\begin{array}{l}\text { pBADHmsF-aa } \\
\text { substitutions }\end{array}$ & $\begin{array}{l}6.9 \mathrm{~kb}, \mathrm{Ap}^{\mathrm{r}} \text {, } \operatorname{araC}^{+} \text {, arabinose-inducible expression of } h m s F \\
\text { with the following substitutions - K107A, D114A, D115A, } \\
\text { DD114/5AA, R123A, W143A, R161A, W167A, Q169A, E172A, } \\
\text { E180A, H184A, Q354A, D358A, D362A, R373A, R384A, W387A, } \\
\text { Q388A, D466A, D467A; all derived from pBADHmsF }\end{array}$ & This study \\
\hline pBADHmsR & $\begin{array}{l}6 \cdot 3 \mathrm{~kb}, \mathrm{Ap}^{\mathrm{r}}, \operatorname{araC}^{+}, \text {arabinose-inducible expression of } h m s R \\
1.4 \mathrm{~kb} \text { PCR fragment generated from pHMS1.2 ligated into } \\
\text { EcoRI-XbaI sites in pBAD30 }\end{array}$ & This study \\
\hline $\begin{array}{l}\text { pBADHmsR-aa } \\
\text { substitutions }\end{array}$ & $\begin{array}{l}6 \cdot 3 \mathrm{~kb}, \mathrm{Ap}^{\mathrm{r}}, \operatorname{araC}^{+} \text {, arabinose-inducible expression of } h m s R \\
\text { with the following substitutions - T114A, D176A, D269A, } \\
\text { Q305A, R308A, L324A; all derived from pBADHmsR }\end{array}$ & This study \\
\hline pBSSK $\Delta$ hmsH & $\begin{array}{l}5 \cdot 3 \mathrm{~kb}, \mathrm{Ap}^{\mathrm{r}}, 1 \cdot 15 \mathrm{~kb} N \text { si }-X m n \mathrm{I} \text { and } S w a \mathrm{I}-\text { Bam HI fragments } \\
\text { from pNPM22 ligated into PstI-BamHI sites of pBluescript SK }\end{array}$ & This study \\
\hline pHmsH-aa substitutions & $\begin{array}{l}9 \cdot 9 \mathrm{~kb}, \mathrm{Cm}^{\mathrm{r}}, h m s H^{+} h m s F^{\prime} \text { expressing } h m s H \text { with the } \\
\text { following substitutions - R143A, E269A, E281A, D289A, } \\
\text { A350V; all derived from pNPM22 }\end{array}$ & This study \\
\hline pKNG101 & $6 \cdot 8 \mathrm{~kb}, \mathrm{Sm}^{\mathrm{r}} \mathrm{Suc}^{\mathrm{s}}\left(\operatorname{sacB}^{+}\right), \mathrm{R} 6 \mathrm{~K}$ origin suicide vector & Kaniga et al. (1991) \\
\hline $\mathrm{pKNG} \Delta \mathrm{hmsH}$ & $\begin{array}{l}9 \cdot 2 \mathrm{~kb}, \mathrm{Sm}^{\mathrm{r}}, 2 \cdot 4 \mathrm{~kb} \text { SalI-XbaI fragment from } \\
\text { pBSSK } \Delta \mathrm{hmsH} \text { ligated into SalI-XbaI sites pKNG101 }\end{array}$ & This study \\
\hline pKNG $\Delta$ hmsR & $\begin{array}{l}9 \cdot 0 \mathrm{~kb}, \mathrm{Sm}^{\mathrm{r}}, 2 \cdot 2 \mathrm{~kb} \text { SalI-XbaI fragment from } \\
\text { pWSK } \Delta \mathrm{hmsR} \text { ligated into SalI-XbaI sites pKNG101 }\end{array}$ & This study \\
\hline pNPM11 & $9 \cdot 3 \mathrm{~kb}, \mathrm{Cm}^{\mathrm{r}}$, encodes intact $h m s F, h m s R$ and $h m s S$ genes & Pendrak \& Perry $(1991,1993)$ \\
\hline pNPM22 & $9 \cdot 9 \mathrm{~kb}, \mathrm{Cm}^{\mathrm{r}}, h m s H^{+} h m s \mathrm{~F}^{\prime}$ & Lillard et al. (1997); Pendrak \& Perry (1993) \\
\hline pNPM38 & $23 \mathrm{~kb}, \mathrm{Km}^{\mathrm{r}} \mathrm{Cm}^{\mathrm{r}}, h m s H F R^{+}, h m s S::$ MudIII1734-38 & Lillard et al. (1997); Pendrak \& Perry (1993) \\
\hline pWSK29 & $5 \cdot 4 \mathrm{~kb}, \mathrm{Ap}^{\mathrm{r}}, \mathrm{Km}^{\mathrm{r}}$, low-copy-number cloning vector & Wang \& Kushner (1991) \\
\hline pWSK $\Delta$ hmsR & $\begin{array}{l}7 \cdot 6 \mathrm{~kb}, \mathrm{Ap}^{\mathrm{r}}, 1 \cdot 1 \mathrm{~kb} \text { SalI-NcoI and } 1 \cdot 1 \mathrm{~kb} \text { BsrBI-BamHI } \\
\text { fragments ligated into SalI-BamHI sites of pWSK29 }\end{array}$ & This study \\
\hline
\end{tabular}

${ }^{\star}$ All Y. pestis strains are avirulent from the absence of the low-calcium response virulence plasmid pCD1 (Lcr ${ }^{-}$). Y. pestis strains with a plus sign possess an intact $102 \mathrm{~kb}$ pgm locus.

$\dagger \mathrm{Ap}^{\mathrm{r}}, \mathrm{Cm}^{\mathrm{r}}, \mathrm{Km}^{\mathrm{r}}$ and $\mathrm{Sm}^{\mathrm{r}}$, resistance to ampicillin, chloramphenicol, kanamycin and streptomycin, respectively. 
pestis KIM6(pKD46) +. Electrocompetent cells were made as previously described (Fetherston et al., 1995) from cultures grown at $26^{\circ} \mathrm{C}$ in $\mathrm{HIB}$ to $\mathrm{OD}_{620} \approx 0.6$ and then incubated for $1.5 \mathrm{~h}$ with $0.2 \%$ arabinose to induce expression of the Red recombinase (Datsenko \& Wanner, 2000). Gene-specific primers (see Table S1, available as supplementary material with the online version of this paper) were used to amplify the cam gene cassette from pKD3. Cells, transformed with $0 \cdot 5-2 \mu \mathrm{g}$ purified PCR product, were plated on TBA plates containing $\mathrm{Cm}$ to select for recombinant cells. To cure pKD46 from selected recombinants, colonies, grown at $37^{\circ} \mathrm{C}$ on TBA without Ap, were isolated and screened for sensitivity to Ap. For selected mutants, the presence of the cam cassette was confirmed by PCR. Flippaseexpressing pCP20 was electroporated into selected $\mathrm{Cm}^{\mathrm{r}}$ mutants and the transformation mixture was plated, after overnight incubation at room temperature, on TBA plates containing Ap. As described above, cells sensitive to $\mathrm{Cm}$ and Ap (excised $\mathrm{Cm}$ cassette and pCP20-cured) were isolated. Mutations were confirmed by PCR and the resulting strains designated KIM6-2116 ( $\Delta h m s F-2116)$ and KIM6-2119 ( $\Delta h m s S-$ 2119). These non-polar mutations remove all of the coding region for the HmsF protein (673 aa) and 150 of 155 aa residues in the HmsS protein. The $h m s F$ deletion in KIM6-2116 overlaps with $h m s R$ and potentially removes four $\mathrm{N}$-terminal amino acids of HmsR. Despite this elimination of the predicted start site (Lillard et al., 1997), expression of this version of HmsR is functional (see Results).

Construction of alanine substitutions in HmsH, HmsF, HmsR and HmsS. Most of the amino acid substitutions were made by a two-step PCR cloning scheme (Fig. 1) using two outside primers $(1,2)$ and two overlapping interior primers $(3,4)$ carrying the desired mutation. This method has been used to avoid the occurrence of PCR mutations while amplifying a long template even with

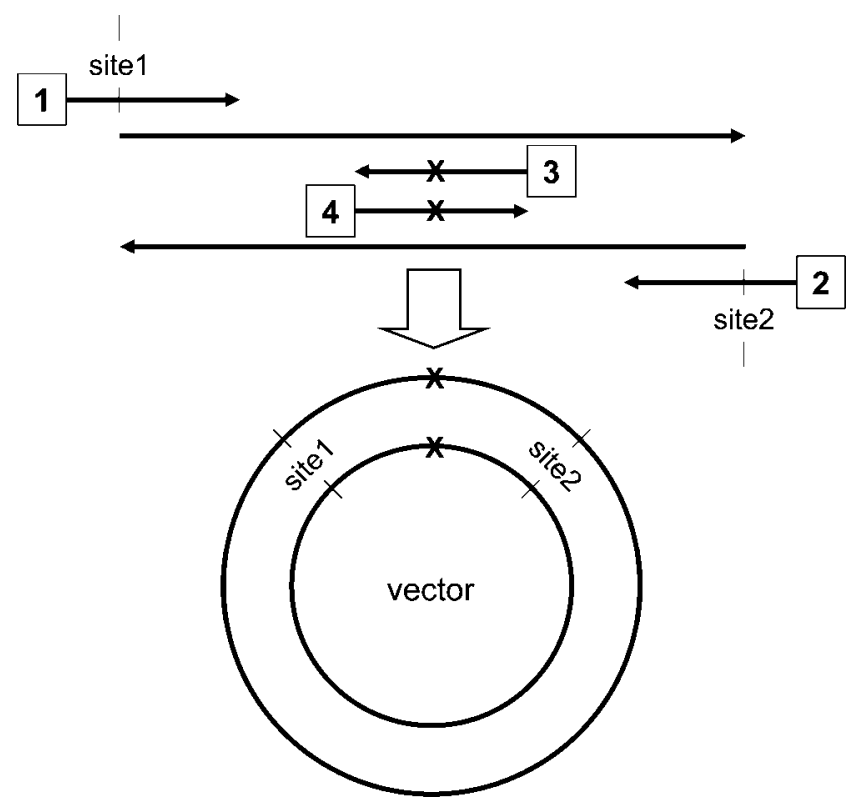

Fig. 1. Diagram of the two-step PCR-substitution. DNA primers 3 and 4 span the region of interest and carry an appropriate mutation (' $X$ ' represents altered sequence yielding an amino acid substitution). Oligonucleotides 1 and 2, incorporating restriction sites 1 and 2, were used to clone the mutated gene into an expression vector with an inducible or native promoter.
DNA polymerases with proof-reading activity. First, two smaller PCR products were generated (primers 1-3 and 4-2), overlapping at the region of interest by primers carrying the desired mutation (primers 3 and 4). PCR products were gel purified, with the exception of $h m s R$ mutations, where a $D p n$ I digestion was used to eliminate carryover template DNA. Second, the products of the first PCR (1-3, 4-2), hybridizing across the site of interest and with the introduced amino acid codon substitution (3-4), underwent a fill-in cycle to yield a full-length (1-2) double-stranded template. Third, the outside primers $(1,2)$ were added to the reaction to complete the second PCR and thus generate a gene fragment carrying the desired mutation. The fill-in PCR consisted of one 2 min cycle at $95^{\circ} \mathrm{C}$ followed by two cycles at $95^{\circ} \mathrm{C}$ for $30 \mathrm{~s}, 50^{\circ} \mathrm{C}$ for $30 \mathrm{~s}$ and $68^{\circ} \mathrm{C}$ for $4 \mathrm{~min}$. After introducing the outside primers $(1,2)$, the PCR reaction continued with a 2 min cycle at $95^{\circ} \mathrm{C}$ followed by 30 cycles at $95^{\circ} \mathrm{C}$ for $30 \mathrm{~s}, 50^{\circ} \mathrm{C}$ for $30 \mathrm{~s}$ and $68^{\circ} \mathrm{C}$ for $4 \mathrm{~min}$. A 4 min extension time was applied in the case of $\mathrm{a} \approx 2 \mathrm{~kb}$ fragment $(h m s F)$ and was modified accordingly for fragments of different sizes. The final PCR product was column purified and concentrated (Zymo Research), digested (site1, site2 in Fig. 1) and cloned into a target vector. ProofStart DNA polymerase (Qiagen) was used in most amplification reactions, unless stated otherwise. In some cases the QuickChange site-directed mutagenesis kit (Stratagene) was used, following the manufacturer's instructions. Sequences of primers specific for each amino acid substitution are given in Table S1.

Mutations in $h m s H$ were constructed in pNPM22 by insertion of modified $h m s H$ PCR fragments digested with $B l p \mathrm{I}$ and $X h o$ I for the $\mathrm{HmsH}-\mathrm{R} 134 \mathrm{~A}$ change and with $\mathrm{BlpI}$ and $\mathrm{Xmn \textrm {I }}$ for $\mathrm{HmsH}$ changes E269A, E281A, D289A and A350V. These recombinant plasmids (e.g. pHmsH-E269A; Table 1) express $h m s H$ genes and a truncated $h m s F$ from the native hmsHFRS promoter.

Plasmid pNPM11 was digested with SwaI/TatI to subclone $h m s F$ into the KpnI-SmaI sites of pBAD30. An isolated EcoRI-XbaI fragment from this plasmid containing the $h m s F$ gene was treated with DNase I (long Klenow fragment) and cloned into the SmaI site of pBAD30, yielding pBADHmsF. All $\mathrm{HmsF}$ substitutions were constructed in pBAD30 by insertion of the modified $h m s F$ PCR fragments generated from pBADhmsF and digested with EcoRI and $\mathrm{XbaI}$ (Table 1).

Plasmid pHMS1.2 was used as a template to amplify wild-type $h m s R$ and $h m s S$ DNA. Both genes were cloned as EcoRI-XbaI fragments into the same sites in pBAD30, generating pBADHmsR and pBADHmsS (Table 1). HmsR D176A and D269A mutations were constructed with the QuickChange site-directed mutagenesis kit (Stratagene). Platinum Pfx DNA polymerase (Invitrogen) was used in the course of $h m s R$ mutagenesis. Substitutions in $h m s S$ were constructed in pBAD30 by insertion of the modified $h m s S$ PCR fragments generated from pBADhmsS and digested with EcoRI and XbaI (Table 1).

Sequence analysis. Amino acid sequences presented in protein alignments (Fig. 2) were obtained from the NCBI Entrez Genome Project website. All sequence alignments were generated in T-Coffee (version 1.6; http://www.ch.embnet.org/software/TCoffee.html/ inter-ref $>$ ) (Notredame et al., 2000).

Western blot analysis. Equal protein concentrations were separated on polyacrylamide gels containing SDS prior to Western blotting onto PVDF membranes (Immobilon P; Millipore). For detection of HmsR, samples were not boiled. A modified Towbin procedure (Towbin et al., 1979) was used for immunodetection. Briefly, PVDF membranes were blocked with $5 \%$ non-fat dry milk or $1.5 \% \mathrm{BSA}$ in $10 \mathrm{mM}$ Tris/ $\mathrm{HCl}(\mathrm{pH} 7 \cdot 6), 137 \mathrm{mM} \mathrm{NaCl}$ (TBS) with $0 \cdot 1 \%$ Tween 20 (TBST) and then incubated with the appropriate antibody diluted in TBST. Antibodies, raised against portions of $\mathrm{HmsH}, \mathrm{HmsF}$ and $\mathrm{HmsS}$ proteins, were generated in rabbits by Animal Pharm Services. Antiserum against a synthetic immunogenic 

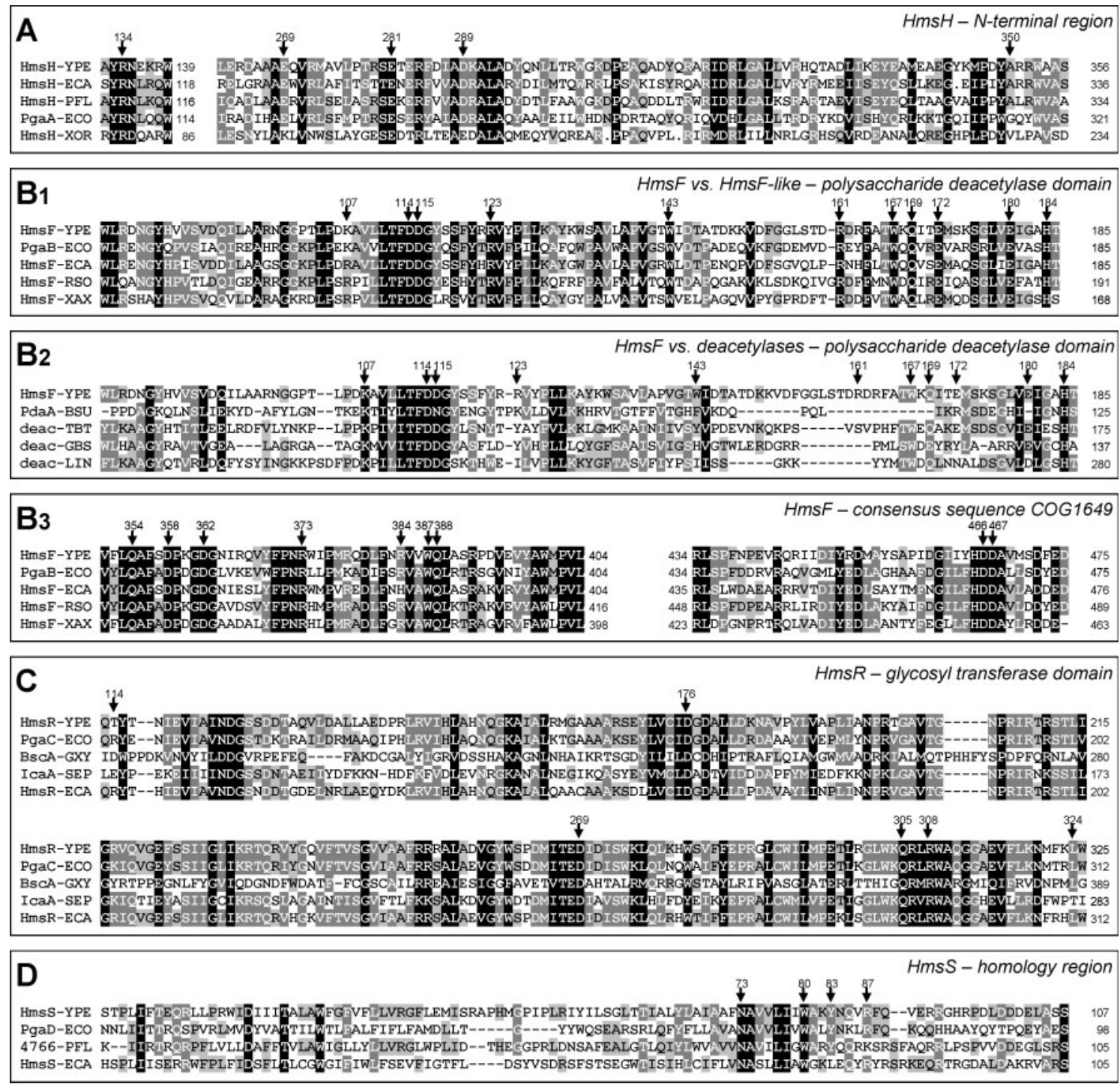

Fig. 2. Alignment of the amino acid sequences in conserved regions of $Y$. pestis $\mathrm{HmsH}(A)$, deacetylase $\left(B_{1}\right.$ and $\left.B_{2}\right)$ and COG1649 (B3) domains of HmsF, HmsR (C), and HmsS (D). All alignments were generated in T-Coffee (version 1.6). The black and grey boxes represent residue identity and similarity, respectively. Residue numbering of $\mathrm{Hms}$ proteins begins with the putative initiating methionine. Arrows indicate residues altered to alanines in $Y$. pestis Hms proteins. The $E$. coli pgaABCD genes encoding proteins involved in biofilm formation (Wang et al., 2004) were originally annotated as ycdSRQP (Blattner et al., 1997). Names of organisms were abbreviated as follows: Erwinia carotovora, ECA; Pseudomonas fluorescens, PFL; Escherichia coli, ECO; Xanthomonas oryzae, XOR; Bacillus subtilis, BSU; Thermoanaerobacter tengcongensis, TBT; Geobacter sulfurreducens, GBS; Leptospira interrogans, LIN; Ralstonia solanacearum, RSO; Xanthomonas axonopodis, XAX; Gluconobacter xylinus, GXY; Staphylococcus epidermidis, SEP.

peptide of HmsR was generated by Research Genetics. Following incubation with horseradish peroxidase-conjugated protein A (Amersham Pharmacia Biotech), the immunoreactive proteins were detected with the ECL enhanced chemiluminescence Western blotting detection reagent (Amersham Pharmacia Biotech). Alternatively, proteins interacting with alkaline phosphatase-labelled anti-rabbit IgG molecules were detected with the chemiluminescent substrate CSPD (Roche). Immunoblot results were visualized on Kodak Biomax light film.

\section{RESULTS}

All four hmsHFRS operon-encoded proteins have orthologues in E. coli, proteins PgaABCD (formerly YcdSRQP), and other Gram-negative bacteria. The amino acid substitutions in the Hms proteins constructed and tested in this study were selected from regions of high sequence similarity comprising identified domains or regions with potential 
enzymic activities (Fig. 2). To test the effect of these substitutions, in-frame deletions of individual $h m s$ genes in $Y$. pestis were constructed. These mutations yielded an $\mathrm{Hms}^{-}$phenotype at $26-30{ }^{\circ} \mathrm{C}$ : white colonies on $\mathrm{CR}$ plates and absence of aggregation in liquid broth cultures. The four cloned wild-type hms genes complemented their respective in-frame or non-polar chromosomal mutations. Variants of $h m s$ genes with single amino acid substitutions in pBAD30 with arabinose-inducible expression ( $h m s F, h m s R$ and $h m s S$ ) or in versions of pNPM22 (e.g. pHmsH-R134A) with expression of $h m s H$ alleles from the native $h m s H$ promoter were tested for the ability to bind CR or to attach to glass in CV-staining assays as parameters of biofilm formation. The cellular level of wild-type and variant $\mathrm{Hms}$ proteins was assessed by Western blot analysis (Table 2).

\section{HmsH and HmsF amino acid substitutions}

$\mathrm{HmsH}$ and $\mathrm{HmsF}$ are proven outer-membrane proteins of $Y$. pestis (Pendrak \& Perry, 1991, Pendrak \& Perry, 1993; Perry et al., 2004) and have amino acid sequence identities/ similarities of $41 \cdot 1 \% / 58 \cdot 2 \%$ and $48 \cdot 3 \% / 60 \cdot 8 \%$, respectively, to E. coli proteins PgaA and $\mathrm{PgaB}$ (Fig. 2). $\mathrm{HmsH}$ is the largest Hms protein (822 aa) with a predicted $\beta$-barrel structure typical of a transmembrane protein in the outer membrane. We have changed charged residues R134, E269, E281 and $\mathrm{D} 289$ to alanines in the $\mathrm{N}$-terminal portion of $\mathrm{HmsH}$. These residues are within a periplasmic domain predicted by the hidden Markov model (PRED_TMBB; http://bioinformatics. biol.uoa.gr/PRED-TMBB). Alanine substitutions E269A and D289A in $\mathrm{HmsH}$ had no significant effect on the Hms phenotype. The E281A change, where the stability of the resulting HmsH-E281A protein was affected (Table 2), apparently complemented the $\Delta h m s H$ mutation on CR plates but gave significantly decreased values in our quantitative assays for $\mathrm{CR}$ adsorption and $\mathrm{CV}$ staining (Fig. 3A, B) compared to complementation with wild-type $h m s H$. The HmsH-E281A protein was defective in biofilm formation as assessed by CV staining (Fig. 3). The $\mathrm{HmsH}-$ $\mathrm{R} 134 \mathrm{~A}$ protein also appears defective in biofilm formation by the CV staining assay but failed to show a similar defect in quantitative CR binding. A neutral change, $h m s H-A 350 \mathrm{~V}$, fully complemented the $\Delta h m s H$ mutation as expected (Fig. 3).

HmsF shows highest overall similarity to a number of HmsF-like proteins of unproven function or enzymic activity (Fig. $2 \mathrm{~B}_{1}$ and $2 \mathrm{~B}_{3}$ ). Several of the conserved residues in this group are not highly conserved in enzymes with proven deacetylase activity from a wide variety of organisms. Comparison of the deacetylase domains of these proteins, including Bacillus subtilis PdaA with known crystal structure (Blair \& van Aalten, 2004), to Y. pestis $\mathrm{HmsF}$ (Fig. 2B $\mathrm{B}_{2}$ ) indicated highly conserved residues related to enzymic activity. The highly conserved nature of all these residues (Fig. $2 \mathrm{~B}_{1-3}$ ) suggests they might be essential.

The D114A alteration in HmsF, corresponding to the D73 residue of $\mathrm{PdaA}$, abolished the $\mathrm{HmsF}$ protein function,
Table 2. Effect of amino acid substitutions in HmsHFRS proteins on the Hms phenotype and protein functionality

\begin{tabular}{|c|c|c|c|}
\hline \multirow[t]{2}{*}{ Gene-aa substitution } & \multirow[t]{2}{*}{ Hms phenotype ${ }^{\star}$} & \multicolumn{2}{|c|}{ Protein } \\
\hline & & Functional & Levels $\dagger$ \\
\hline$h m s H-w t$ & Red & Yes & - \\
\hline$h m s H-R 134 A$ & Red & Yes & wt \\
\hline$h m s H-E 269 A$ & Red & Yes & wt \\
\hline$h m s H-E 281 A$ & Red & Partially & $\ddagger$ \\
\hline$h m s H-D 289 A$ & Red & Yes & wt \\
\hline$h m s H-A 350 \mathrm{~V}$ & Red & Yes & wt \\
\hline$h m s F-w t$ & Red & Yes & - \\
\hline \multicolumn{4}{|c|}{ HmsF deacetylase domain } \\
\hline$h m s F-K 107 A$ & Red & Yes & wt \\
\hline$h m s F-D 114 A$ & White & No & wt \\
\hline$h m s F-D 115 A$ & White & No & wt \\
\hline$h m s F-D D 114 / 5 A A$ & White & No & wt \\
\hline$h m s F-R 123 A$ & Red & Yes & wt \\
\hline$h m s F-W 143 A$ & Pink & Partially & wt \\
\hline$h m s F-R 161 A$ & Red & Yes & wt \\
\hline$h m s F-W 167 A$ & Red & Yes & wt \\
\hline$h m s F-Q 169 A$ & Red & Yes & wt \\
\hline$h m s F-E 172 A$ & Red & Yes & wt \\
\hline$h m s F-E 180 A$ & Red & Yes & wt \\
\hline$h m s F-H 184 A$ & White & No & wt \\
\hline \multicolumn{4}{|c|}{ HmsF COG1649 domain } \\
\hline$h m s F-Q 354 A$ & Red & Yes & wt \\
\hline$h m s F-D 358 A$ & Red & Yes & wt \\
\hline$h m s F-D 362 A$ & Red & Yes & wt \\
\hline$h m s F-R 373 A$ & Red & Yes & wt \\
\hline$h m s F-R 384 A$ & Red & Yes & wt \\
\hline$h m s F-W 387 A$ & Red & Yes & wt \\
\hline$h m s F-Q 388 A$ & Red & Yes & wt \\
\hline$h m s F-D 466 A$ & Red & Yes & wt \\
\hline$h m s F-D 467 A$ & Red & Yes & wt \\
\hline$h m s R-w t$ & Red & Yes & - \\
\hline$h m s R-T 114 A$ & Red & Yes & wt \\
\hline$h m s R-D 176 A$ & White & No & wt \\
\hline$h m s R-D 269 A$ & White & No & wt \\
\hline hmsR-Q305A & White & No & wt \\
\hline$h m s R-R 308 A$ & White & No & wt \\
\hline$h m s R-L 324 A$ & Red & Yes & wt \\
\hline$h m s S-w t$ & Red & Yes & - \\
\hline$h m s S-N 73 A$ & Red & Yes & wt \\
\hline$h m s S-W 80 A$ & White & No & wt \\
\hline$h m s S-Y 83 A$ & White & No & $\ddagger$ \\
\hline$h m s S-R 87 A$ & Red & Yes & wt \\
\hline
\end{tabular}

${ }^{*} \mathrm{CR}$ binding assessed as red to white colonies on $\mathrm{CR}$ plates incubated at $26^{\circ} \mathrm{C}$.

$\dagger w t$, variant protein level same as wt protein level.

$\ddagger$ Variant protein not detected by Western blot analysis.

confirming the importance of this residue in the deacetylase domain. Moreover, the D115A substitution in the 'TFD(D)G' 

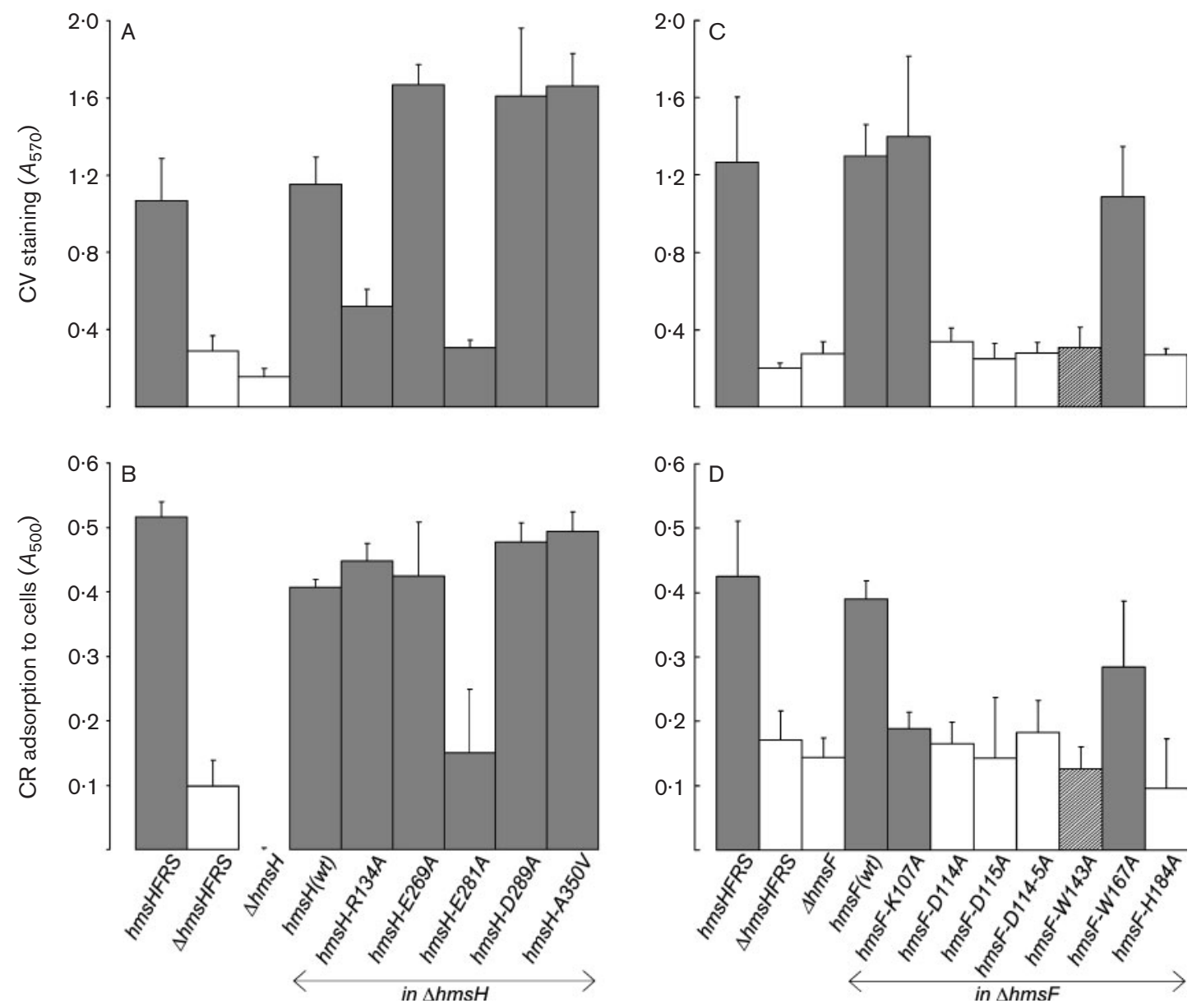

Fig. 3. Analysis of $C V$ staining and $C R$ binding of $Y$. pestis cells expressing amino acid substitutions in $\mathrm{HmsH}(\mathrm{A}$ and $\mathrm{B})$ and $\mathrm{HmsF}\left(\mathrm{C}\right.$ and D) proteins. CV staining of cells attached to borosilicate test tubes after growth at $26{ }^{\circ} \mathrm{C}(\mathrm{A}$ and $\mathrm{C})$ and quantitative $\mathrm{CR}$ binding by $Y$. pestis cells after $3 \mathrm{~h}$ incubation at $26^{\circ} \mathrm{C}$ with $\mathrm{CR}\left(\mathrm{B}\right.$ and D). Strains: $\mathrm{KIM} 6+\left(h m s H F R S^{+}\right)$,

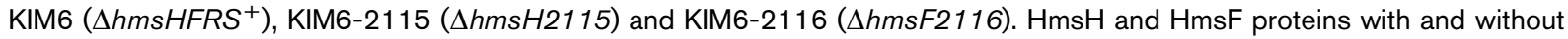
amino acid substitutions were expressed from pNPM22 derivatives and pBADHmsF plasmids, respectively. Grey and open bars indicate formation of $\mathrm{CR}^{+}$and $\mathrm{CR}^{-}$colonies on $\mathrm{CR}$ plates, respectively. The hatched bar indicates formation of an intermediate pinkish colony on CR plates. Data shown represent a mean of at least four independent experiments carried out with sample duplicates. Displayed error bars refer to standard deviation values.

conserved motif and alteration of the most conserved $\mathrm{H} 184$ residue in $\mathrm{HmsF}$ both caused biofilm-defective phenotypes (Fig. 3C, D). HmsF with a double substitution (D114A and D115A) retained the expected phenotypic loss as well. Another strongly conserved residue among proven deacetylases, K107, was not critical for $\mathrm{HmsF}$ function (Fig. 3C, D). However, the K107A change caused an 50\% loss in CR binding. Among the residues conserved in $\mathrm{HmsF}-$ like proteins (R123, W143, R161, W167, Q169, E172 and E180), only the W143 substitution had an effect on HmsF function (Table 2). The W143A substitution (identical to W143 of E. coli PgaB but not present in other deacetylases) had an intermediate-pink phenotype on CR agar plates (Table 2) and an adverse effect in both quantitative assays we used to assess the Hms phenotype (Fig. 3C, D).
The C-terminal part of $Y$. pestis $\mathrm{HmsF}$ has a predicted domain COG1649 that is present in other HmsF-like proteins of Gram-negative bacteria (Fig. $2 \mathrm{~B}_{2}$ ). None of the nine individual substitutions in conserved residues in the COG1649 region of $\mathrm{HmsF}$ caused a biofilm-defective phenotype (Table 2). An altered HmsF protein with a large deletion (aa 374-674) in COG1649 failed to complement our $\Delta h m s F$ mutant. However, Western blots did not detect the truncated protein, indicating that either the stability of the protein was affected or our polyclonal antibody against HmsF does not detect the truncated protein (data not shown).

\section{HmsR and HmsS amino acid substitutions}

The similarities of the putative glycosyltransferase HmsR from Y. pestis to those of Staphylococcus epidermidis - IcaA 
and E. coli - PgaC (formerly YcdQ) and to the cellulose synthetase BscA from Salmonella typhimurium have been described previously (Jones et al., 1999; Kirillina et al., 2004; Lillard et al., 1997; Wang et al., 2004). The functionality of these proteins is critical for biofilm formation in all these bacteria (Heilmann et al., 1996; Lillard et al., 1997; Pendrak \& Perry, 1993; Wang et al., 2004; Zogaj et al., 2001). Amino acid sequence comparison of the cellulose synthase BscA from Gluconobacter xylinus with many other putative glycosyltransferases, including $\mathrm{HmsR}$, IcaA and PgaC, led to the identification of a conserved ' $\mathrm{D}, \mathrm{D}, \mathrm{D} 35 \mathrm{QxxRW}$ ' motif (Saxena \& Brown, 1997) (Fig. 2C). We therefore tested alanine substitutions at positions D176, D269, Q305 and R308 as well as in non-conserved residues T114 and L324. Expression of HmsR-T114A and HmsR-L324A complemented the $\Delta h m s R$ mutation as well as the wildtype $h m s R$ gene in forming red colonies on CR agar and restored high levels of CR binding and CV staining (Fig. 4). However, substitutions D176A, D269A, Q305A or R308A in HmsR did not complement the $\Delta h m s R$ mutant, giving rise to white colonies on CR plates and only low levels of CV staining. For all $\mathrm{HmsR}$ variants tested, the results of the quantitative CR-binding assays correlated with CV-staining assays (Fig. 4A, B). Hence, four conserved residues from the glycosyltransferase motif ' $\mathrm{D}, \mathrm{D}, \mathrm{D} 35 \mathrm{QxxRW}$ ' in HmsR, D176, D269, R305, R308, are critical for production of the biofilm extracellular matrix.

HmsS $(17.5 \mathrm{kDa})$ is the smallest Hms protein in Y. pestis, with two predicted transmembrane domains but no other identified domains. A ' $\mathrm{NA}(\mathrm{V}) \mathrm{xLIxW}(\mathrm{A}) \mathrm{x}(\mathrm{Y}) \mathrm{x}(\mathrm{Q}) \mathrm{x}(\mathrm{R})$ ' sequence is highly conserved in E. coli PgaD, Erwinia carotovora $\mathrm{HmsS}$ and Pseudomonas fluorescens $\mathrm{HmsS}$ (Fig. 2D) and was selected for mutagenesis. Expression of
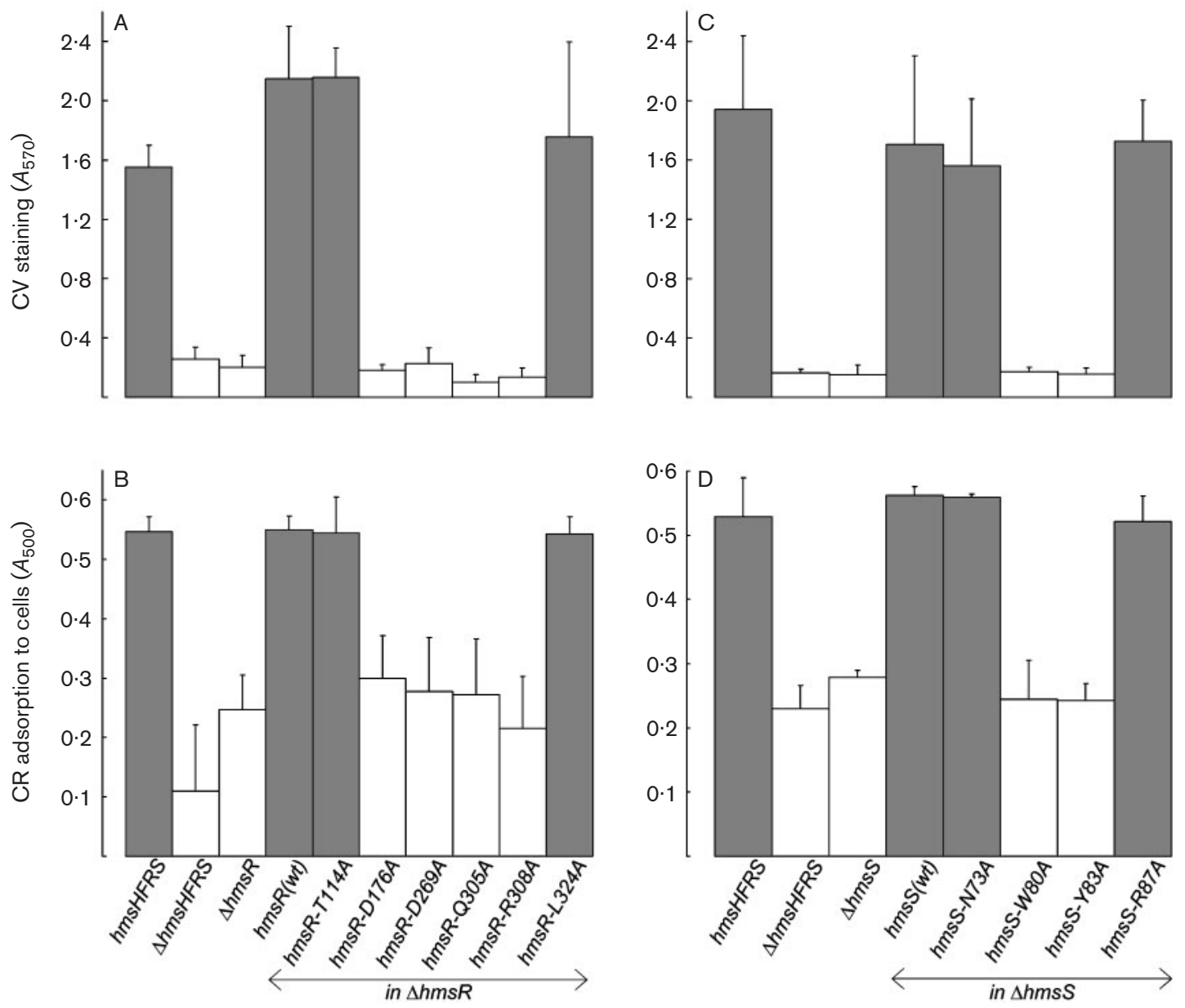

Fig. 4. Analysis of $C V$ staining and $C R$ binding of $Y$. pestis cells expressing amino acid substitutions in $H m s R(A$ and $B)$ and $\mathrm{HmsS}(C$ and $D)$ proteins. CV staining of cells attached to borosilicate test tubes after growth at $26^{\circ} \mathrm{C}(\mathrm{A}$ and $\mathrm{C})$ and quantitative $\mathrm{CR}$ binding by $Y$. pestis cells after $3 \mathrm{~h}$ incubation at $26^{\circ} \mathrm{C}$ with $\mathrm{CR}\left(\mathrm{B}\right.$ and D). Strains: $\mathrm{KIM6}+\left(h m s H F R S^{+}\right)$,

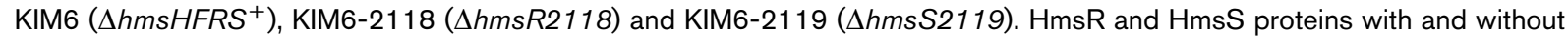
amino acid substitutions were expressed from pBADHmsR and pBADHmsS plasmids, respectively. Grey and open bars indicate formation of $\mathrm{CR}^{+}$and $\mathrm{CR}^{-}$colonies on $\mathrm{CR}$ plates, respectively. Data shown represent a mean of at least two (HmsS: three) independent experiments carried out with sample duplicates. Displayed error bars refer to standard deviation values. 
HmsS-N73A or HmsS-R87A in KIM6-2119 ( $\Delta h m s S)$ restored $\mathrm{CR}$ binding and biofilm formation. On the other hand, substitutions W80A and Y83A gave a strong biofilmdefective phenotype equal to that of KIM6 ( $\triangle h m s H F R S$ ), with little CV staining or CR binding. Thus, the W80A and Y83A, but not the N73A and R87A, residues are critical to the function of HmsS.

\section{DISCUSSION}

Previously, we used transposon insertion mutants and complementation to conclude that all four genes in the hmsHFRS operon are essential for CR binding and thus biofilm formation (Lillard et al., 1997; Pendrak \& Perry, 1993). In this study, we constructed non-polar deletion mutations in each gene of the operon and demonstrated loss of biofilm formation in each mutant via quantitative CR-binding and CV-staining assays. Moreover, biofilm formation was restored by complementation with the corresponding gene (Figs 3 and 4). This definitively confirms our previous conclusions that $\mathrm{HmsH}$, $\mathrm{HmsF}$, $\mathrm{HmsR}$ and $\mathrm{HmsS}$ are each essential for biofilm formation.

Genes encoding orthologues of each of these Y. pestis proteins have been identified in a number of diverse organisms (Bell et al., 2004; da Silva et al., 2002; Jones et al., 1999; Lee et al., 2005; Paulsen et al., 2005; Salanoubat et al., 2002; Wang et al., 2004). Of these, the E. coli PgaABCD system is involved in formation of an EPS containing a $\beta$ 1,6- $N$-acetyl-D-glucosamine polymer, and an EPS with a similar component is also part of the Y. pestis biofilm (Itoh et al., 2005; Wang et al., 2004). Several other proteins related to $\mathrm{HmsH}, \mathrm{HmsF}, \mathrm{HmsR}$ and $\mathrm{HmsS}$ are from genome sequence projects (Bao et al., 2002; Methé et al., 2003; Nascimento et al., 2004; Ren et al., 2003). We have used these in amino acid sequence alignments with the Hms proteins to identify highly conserved residues that might be critical to the function of these four $\mathrm{Hms}$ proteins. Alteration of selected conserved residues to alanine identified critical residues, residues with a moderate effect, and highly conserved residues that are not important for forming a functional Hms protein.

$\mathrm{HmsH}$ is predicted to form a $\beta$-barrel protein with a large $\mathrm{N}$ terminal periplasmic domain (Bagos et al., 2004; Kirillina et al., 2004). Our mutagenesis study concentrated on this periplasmic domain to avoid a loss of phenotype due to disruption of the $\beta$-barrel structure. Two of four residues (E269 and D289) had no effect on biofilm formation while the other two (R134 and E281) had only a moderate effect on the composition of the usual Y. pestis KIM6 + biofilm. Complementation with both mutated $\mathrm{HmsH}$ proteins yielded $\mathrm{CR}^{+}$colonies on $\mathrm{CR}$ plates, indicating no change in a long-term phenotype ( $\mathrm{CR}$ binding over $>48 \mathrm{~h}$ ). However, both HmsH-E281A and HmsH-R134A had a significant reduction in $\mathrm{CV}$ staining compared to $\mathrm{HmsH}$, indicating an alteration in biomass bound to glass over a period of $\sim 16 \mathrm{~h}$. HmsH-E281A also caused a reduction in
CR binding in liquid assay over a $3 \mathrm{~h}$ period. Thus, R134 or E281 of HmsH are not absolutely crucial to the function of $\mathrm{HmsH}$ but may moderately reduce its efficacy.

HmsF belongs to a large family of deacetylases that possess strong sequence similarities across a wide range of bacteria, with some variability due to the distinct carbohydrate substrates used. The role of $B$. subtilis PdaA in spore germination has been described in detail and in vitro enzymic studies have demonstrated PdaA deacetylase activity with an $\mathrm{N}$-acetylmuramic acid substrate (Fukushima et al., 2002, 2005; Gilmore et al., 2004). PdaB deacetylase is involved in assembly of peptidoglycan during the course of B. subtilis sporulation (Fukushima et al., 2004). Within the HmsF deacetylase domain, we selected 11 highly conserved residues to mutate. Four residues are highly conserved in proven deacetylases (Fig. $2 \mathrm{~B}_{2}$ ) while the other seven residues are highly conserved in HmsF-like proteins (Fig. $2 \mathrm{~B}_{1}$ ) but not in proven deacetylases (Fig. $2 \mathrm{~B}_{2}$ ). Alteration of the HmsF residues D114 and D115, corresponding to D73 and N74 of PdaA, completely prevented biofilm formation, possibly by affecting the putative deacetylase activity of $\mathrm{HmsF}$. The H184A substitution (corresponding to H124 in PdaA) also abolished CR binding and CV staining. However, the HmsF containing the K107A alteration appears to produce another attenuated CRbinding mutant with reduced short-term binding at $3 \mathrm{~h}$ that does not affect the formation of CR colonies by $48 \mathrm{~h}$ of growth (Fig. 3, Table 2). The W143A substitution caused negligible $\mathrm{CR}$ binding and $\mathrm{CV}$ straining that led to an intermediate-pink colony formation on CR plates, suggesting this residue is not essential but does affect protein function - possibly enzymic activity. W143 was the only substitution in HmsF-like conserved residues that had any effect on the functionality of HmsF.

A second region of $Y$. pestis $\mathrm{HmsF}$ with high similarity to other proteins (Fig. $2 \mathrm{~B}_{2}$ ) is COG1649; proteins with this domain of unknown function are assigned to the TIM barrel glycosyl hydrolase superfamily by Pfam (Bateman et al., 2004). Nine alanine substitutions of highly conserved residues within the COG1649 domain of $\mathrm{HmsF}$ did not affect $\mathrm{HmsF}$ - all altered proteins were able to fully restore an $\mathrm{Hms}^{+}$phenotype (Table 2). However, expression of a truncated HmsF lacking the COG1649 domain failed to complement the $\Delta h m s F$ mutant. Truncated HmsF protein was not detected by HmsF antiserum used in immunodetection of full-length $\mathrm{HmsF}$ proteins. The recombinant protein used to produce $\mathrm{HmsF}$ antiserum lacked the first 87 aa but contained both the deacetylase and COG1649 domains (Kirillina et al., 2004; Pendrak \& Perry, 1991; Perry et al., 2004). This may indicate protein instability or lack of antigenic domains recognized by our $\mathrm{HmsF}$ antibody. Further studies will be required to determine whether the COG1649 domain is important to the function of HmsF.

There are several lines of evidence indicating that HmsR is a likely $\beta-1,6-N$-acetyl-D-glucosamine transferase. First, HmsR has a high degree of similarity to IcaA and PgaC, 
which are both required for the synthesis $\beta-1,6-N$-acetyl-Dglucosamine (Heilmann et al., 1996; Jones et al., 1999; Wang et al., 2004). Second, $\mathrm{PgaC}$ was able to restore the CR-binding phenotype of an $h m s R$ mutant when expressed from a highcopy-number plasmid (Jones et al., 1999). Third, DspB, a $\beta$-hexosaminidase and biofilm-dispersing enzyme of $A$. actinomycetemcomitans (Kaplan et al., 2003), shown to specifically hydrolyse the glycosidic linkages of poly- $\beta-N$ acetyl-D-glucosamine, caused complete inhibition of biofilm formation by Y. pestis KIM6 + (Itoh et al., 2005). Our results further support this conclusion. A non-polar hmsR deletion abrogated synthesis of an extracellular matrix which binds CR and expression of HmsR proteins with substitutions in conserved residues of the glycosyltransferase motif ' $D, D$, D35QxxRW', i.e. D176A, D269A, R305A and R308A, failed to restore CR binding and CV staining to this mutant (Fig. 2; Table 2). The substitutions D236Y, D333R, Q369M and R372A within this motif in the AcsAB cellulose synthase of $G$. xylinus impaired the enzymic activity in vitro (Saxena \& Brown, 1997; Saxena et al., 2001). Consequently, we propose that the substitutions in $\mathrm{HmsR}$ at similar positions may abolish its enzymic activity in poly- $N$-acetylglucosamine production.

The essential HmsS protein, which is associated with the inner membrane like HmsR and HmsT (Lillard et al., 1997; Perry et al., 2004), has two predicted transmembrane regions but no other currently recognized domains. Alignment of HmsS-like proteins identified a conserved region ['NA(V)xLIxW(A) $\mathrm{x}(\mathrm{Y}) \mathrm{x}(\mathrm{Q}) \mathrm{x}(\mathrm{R})$ '; Fig. 2D)]. N73A and R87A changes did not affect HmsS protein function. In contrast, a dramatic loss of $\mathrm{HmsS}$ function was related to W80A and Y83A alterations (Fig. 4A, B). Both W80 and Y83 are within a predicted cytoplasmic region of HmsS.

These site-directed mutagenesis studies have identified critical residues within predicted enzymic domains in $\mathrm{HmsF}$ and $\mathrm{HmsR}$ and in a conserved region of $\mathrm{HmsS}$. Two residue changes in $\mathrm{HmsH}$ and two in $\mathrm{HmsF}$ ( $\mathrm{HmsH}-\mathrm{R} 134 \mathrm{~A}$, HmsH-E281, HmsF-K107A, HmsF-W134A) had differential effects in our biofilm assays. The reason for this differential effect is obscure. The ability of cells expressing HmsF-K107A to achieve normal attachment levels while displaying an EPS-defective phenotype suggests that this mutant produces an EPS form that is not conducive to CR binding. In addition, these and some of the other amino acid residue substitution mutations may affect protein-protein interactions that may be important for biofilm formation.

\section{ACKNOWLEDGEMENTS}

This study was supported by Public Health Service grant AI25098 from the US National Institutes of Health.

\section{REFERENCES}

Ausubel, F. M., Brent, R., Kingston, R. E., Moore, D. D., Seidman, J. G., Smith, J. A. \& Struhl, K. (1987). Current Protocols in Molecular Biology. New York: Wiley.
Bacot, A. W. (1915). LXXXI. Further notes on the mechanism of the transmission of plague by fleas. J Hyg 14, 774-776.

Bacot, A. W. \& Martin, C. J. (1914). LXVII. Observations on the mechanism of the transmission of plague by fleas. J Hyg 13, 423-439.

Bagos, P. G., Liakopoulos, T. D., Spyropoulos, I. C. \& Hamodrakas, S. J. (2004). PRED-TMBB: a web server for predicting the topology of $\beta$-barrel outer membrane proteins. Nucleic Acids Res 32, W400-W404.

Bao, Q., Tian, Y., Li, W. \& 18 other authors (2002). A complete sequence of the T. tengcongensis genome. Genome Res 12, 689-700.

Bateman, A., Coin, L., Durbin, R. \& 10 other authors (2004). The Pfam protein families database. Nucleic Acids Res 32, D138-D141.

Bearden, S. W. \& Perry, R. D. (1999). The Yfe system of Yersinia pestis transports iron and manganese and is required for full virulence of plague. Mol Microbiol 32, 403-414.

Bell, K. S., Sebaihia, M., Pritchard, L. \& 29 other authors (2004). Genome sequence of the enterobacterial phytopathogen Erwinia carotovora subsp. atroseptica and characterization of virulence factors. Proc Natl Acad Sci U S A 101, 11105-11110.

Birnboim, H. C. \& Doly, J. (1979). A rapid alkaline extraction procedure for screening recombinant plasmid DNA. Nucleic Acids Res 7, 1513-1523.

Blair, D. E. \& van Aalten, D. M. F. (2004). Structures of Bacillus subtilis PdaA, a family 4 carbohydrate esterase, and a complex with $\mathrm{N}$-acetylglucosamine. FEBS Lett 570, 13-19.

Blattner, F. R., Plunkett, G., III, Bloch, C. A. \& 14 other authors (1997). The complete genome sequence of Escherichia coli K-12. Science 277, 1453-1474.

Bobrov, A. G., Kirillina, O. \& Perry, R. D. (2005). The phosphodiesterase activity of the HmsP EAL domain is required for negative regulation of biofilm formation in Yersinia pestis. FEMS Microbiol Lett 247, 123-130.

Branda, S. S., Vik, Å., Friedman, L. \& Kolter, R. (2005). Biofilms: the matrix revisited. Trends Microbiol 13, 20-26.

Darby, C., Hsu, J. W., Ghori, N. \& Falkow, S. (2002). Caenorhabditis elegans: plague bacteria biofilm blocks food intake. Nature 417, 243-244.

Darby, C., Ananth, S. L., Tan, L. \& Hinnebusch, B. J. (2005). Identification of $g m h A$, a Yersinia pestis gene required for flea blockage, by using a Caenorhabditis elegans biofilm system. Infect Immun 73, 7236-7242.

da Silva, A. C. R., Ferro, J. A., Reinach, F. C. \& 62 other authors (2002). Comparison of the genomes of two Xanthomonas pathogens with differing host specificities. Nature 417, 459-463.

Datsenko, K. A. \& Wanner, B. L. (2000). One-step inactivation of chromosomal genes in Escherichia coli K-12 using PCR products. Proc Natl Acad Sci U S A 97, 6640-6645.

Davey, M. E. \& O'Toole, G. A. (2000). Microbial biofilms: from ecology to molecular genetics. Microbiol Mol Biol Rev 64, 847-867.

Deng, W., Burland, V., Plunkett, G., III \& 18 other authors (2002). Genome sequence of Yersinia pestis KIM. J Bacteriol 184, 4601-4611.

Fetherston, J. D., Schuetze, P. \& Perry, R. D. (1992). Loss of the pigmentation phenotype in Yersinia pestis is due to the spontaneous deletion of $102 \mathrm{~kb}$ of chromosomal DNA which is flanked by a repetitive element. Mol Microbiol 6, 2693-2704.

Fetherston, J. D., Lillard, J. W., Jr \& Perry, R. D. (1995). Analysis of the pesticin receptor from Yersinia pestis: role in iron-deficient growth and possible regulation by its siderophore. J Bacteriol 177, 1824-1833.

Fukushima, T., Yamamoto, H., Atrih, A., Foster, S. J. \& Sekiguchi, J. (2002). A polysaccharide deacetylase gene $(p d a A)$ is required for 
germination and for production of muramic $\delta$-lactam residues in the spore cortex of Bacillus subtilis. J Bacteriol 184, 6007-6015.

Fukushima, T., Tanabe, T., Yamamoto, H., Hosoya, S., Sato, T., Yoshikawa, H. \& Sekiguchi, J. (2004). Characterization of a polysaccharide deacetylase gene homologue $(p d a B)$ on sporulation of Bacillus subtilis. J Biochem 136, 283-291.

Fukushima, T., Kitajima, T. \& Sekiguchi, J. (2005). A polysaccharide deacetylase homologue, PdaA, in Bacillus subtilis acts as an $N$ acetylmuramic acid deacetylase in vitro. J Bacteriol 187, 1287-1292.

Gilmore, M. E., Bandyopadhyay, D., Dean, A. M., Linnstaedt, S. D. \& Popham, D. L. (2004). Production of muramic $\delta$-lactam in Bacillus subtilis spore peptidoglycan. J Bacteriol 186, 80-89.

Guzman, L. M., Belin, D., Carson, M. J. \& Beckwith, J. (1995). Tight regulation, modulation, and high-level expression by vectors containing the arabinose $\mathrm{P}_{\mathrm{BAD}}$ promoter. J Bacteriol 177, 4121-4130.

Hare, J. M. \& McDonough, K. A. (1999). High-frequency RecAdependent and -independent mechanisms of Congo red binding mutations in Yersinia pestis. J Bacteriol 181, 4896-4904.

Hartzell, P. L., Millstein, J. \& LaPaglia, C. (1999). Biofilm formation in hyperthermophilic Archaea. Methods Enzymol 310, 335-349.

Heilmann, C., Schweitzer, O., Gerke, C., Vanittannakom, N., Mack, D. \& Götz, F. (1996). Molecular basis of intercellular adhesion in the biofilm-forming Staphylococcus epidermidis. Mol Microbiol 20, 1083-1091.

Hinnebusch, B. J., Perry, R. D. \& Schwan, T. G. (1996). Role of the Yersinia pestis hemin storage ( $h m s)$ locus in the transmission of plague by fleas. Science 273, 367-370.

Humphreys, G. O., Willshaw, G. A. \& Anderson, E. S. (1975). A simple method for the preparation of large quantities of pure plasmid DNA. Biochim Biophys Acta 383, 457-463.

Itoh, Y., Wang, X., Hinnebusch, B. J., Preston, J. F., III \& Romeo, T. (2005). Depolymerization of $\beta-1,6-N$-acetyl-D-glucosamine disrupts the integrity of diverse bacterial biofilms. J Bacteriol 187, 382-387.

Jarrett, C. O., Deak, E., Isherwood, K. E., Oyston, P. C., Fischer, E. R., Whitney, A. R., Kobayashi, S. D., DeLeo, F. R. \& Hinnebusch, B. J. (2004). Transmission of Yersinia pestis from an infectious biofilm in the flea vector. $J$ Infect Dis 190, 783-792.

Jones, H. A., Lillard, J. W., Jr \& Perry, R. D. (1999). HmsT, a protein essential for expression of the haemin storage $\left(\mathrm{Hms}^{+}\right)$phenotype of Yersinia pestis. Microbiology 145, 2117-2128.

Kaniga, K., Delor, I. \& Cornelis, G. R. (1991). A wide-host-range suicide vector for improving reverse genetics in Gram-negative bacteria: inactivation of the blaA gene of Yersinia enterocolitica. Gene 109, 137-141.

Kaplan, J. B., Meyenhofer, M. F. \& Fine, D. H. (2003). Biofilm growth and detachment of Actinobacillus actinomycetemcomitans. J Bacteriol 185, 1399-1404.

Kirillina, O., Fetherston, J. D., Bobrov, A. G., Abney, J. \& Perry, R. D. (2004). HmsP, a putative phosphodiesterase, and HmsT, a putative diguanylate cyclase, control Hms-dependent biofilm formation in Yersinia pestis. Mol Microbiol 54, 75-88.

Lee, B.-M., Park, Y.-J., Park, D.-S. \& 16 other authors (2005). The genome sequence of Xanthomonas oryzae pathovar oryzae KACC10331, the bacterial blight pathogen of rice. Nucleic Acids Res 33, 577-586.

Lillard, J. W., Jr, Fetherston, J. D., Pedersen, L., Pendrak, M. L. \& Perry, R. D. (1997). Sequence and genetic analysis of the hemin storage (hms) system of Yersinia pestis. Gene 193, 13-21.

Lillard, J. W., Jr, Bearden, S. W., Fetherston, J. D. \& Perry, R. D. (1999). The haemin storage $\left(\mathrm{Hms}^{+}\right)$phenotype of Yersinia pestis is not essential for the pathogenesis of bubonic plague in mammals. Microbiology 145, 197-209.
Methé, B. A., Nelson, K. E., Eisen, J. A. \& 31 other authors (2003). Genome of Geobacter sulfurreducens: metal reduction in subsurface environments. Science 302, 1967-1969.

Nascimento, A. L. T. O., Ko, A. I., Martins, E. A. L. \& 44 other authors (2004). Comparative genomics of two Leptospira interrogans serovars reveals novel insights into physiology and pathogenesis. J Bacteriol 186, 2164-2172.

Notredame, C., Higgins, D. G. \& Heringa, J. (2000). T-coffee: a novel method for fast and accurate multiple sequence alignment. J Mol Biol 302, 205.

O'Toole, G. A., Pratt, L. A., Watnick, P. I., Newman, D. K., Weaver, V. B. \& Kolter, R. (1999). Genetic approaches to study of biofilms. Methods Enzymol 310, 91-109.

Paulsen, I. T., Press, C. M., Ravel, J. \& 26 other authors (2005). Complete genome sequence of the plant commensal Pseudomonas fluorescens Pf-5. Nat Biotechnol 23, 873-878.

Pendrak, M. L. \& Perry, R. D. (1991). Characterization of a heminstorage locus of Yersinia pestis. Biol Metals 4, 41-47.

Pendrak, M. L. \& Perry, R. D. (1993). Proteins essential for expression of the $\mathrm{Hms}^{+}$phenotype of Yersinia pestis. Mol Microbiol 8, 857-864.

Perry, R. D. \& Fetherston, J. D. (1997). Yersinia pestis - etiologic agent of plague. Clin Microbiol Rev 10, 35-66.

Perry, R. D., Pendrak, M. L. \& Schuetze, P. (1990). Identification and cloning of a hemin storage locus involved in the pigmentation phenotype of Yersinia pestis. J Bacteriol 172, 5929-5937.

Perry, R. D., Lucier, T. S., Sikkema, D. J. \& Brubaker, R. R. (1993). Storage reservoirs of hemin and inorganic iron in Yersinia pestis. Infect Immun 61, 32-39.

Perry, R. D., Bobrov, A. G., Kirillina, O., Jones, H. A., Pedersen, L. L., Abney, J. \& Fetherston, J. D. (2004). Temperature regulation of the hemin storage $\left(\mathrm{Hms}^{+}\right)$phenotype of Yersinia pestis is posttranscriptional. J Bacteriol 186, 1638-1647.

Pollitzer, R. (1954). Plague. World Health Organ Monogr Ser 22, $1-698$.

Ren, S.-X., Fu, G., Jiang, X.-G. \& 36 other authors (2003). Unique physiological and pathogenic features of Leptospira interrogans revealed by whole-genome sequencing. Nature 422, 888-893.

Salanoubat, M., Genin, S., Artiguenave, F. \& 25 other authors (2002). Genome sequence of the plant pathogen Ralstonia solanacearum. Nature 415, 497-502.

Sambrook, J. \& Russell, D. W. (2001). Molecular Cloning: a Laboratory Manual, 3rd edn. Cold Spring Harbor, NY: Cold Spring Harbor Laboratory.

Saxena, I. M. \& Brown, R. M., Jr (1997). Identification of cellulose synthase(s) in higher plants: sequence analysis of processive $\beta$ glycosyltransferases with the common motif " $D$, $D$, D35Q(R,Q)XRW". Cellulose 4, 33-49.

Saxena, I. M., Brown, J., Malcolm, R. \& Dandekar, T. (2001). Structure-function characterization of cellulose synthase: relationship to other glycosyltransferases. Phytochemistry 57, 1135-1148.

Simm, R., Fetherston, J. D., Kader, A., Römling, U. \& Perry, R. D. (2005). Phenotypic convergence mediated by GGDEF-domaincontaining proteins. J Bacteriol 187, 6816-6823.

Spiers, A. J., Kahn, S. G., Bohannon, J., Travisano, M. \& Rainey, P. B. (2002). Adaptive divergence in experimental populations of Pseudomonas fluorescens. I. Genetic and phenotypic bases of wrinkly spreader fitness. Genetics 161, 33-46.

Straley, S. C. \& Bowmer, W. S. (1986). Virulence genes regulated at the transcriptional level by $\mathrm{Ca}^{2+}$ in Yersinia pestis include structural genes for outer membrane proteins. Infect Immun 51, 445-454. 
Tan, L. \& Darby, C. (2004). A movable surface: formation of Yersinia sp. biofilms on motile Caenorhabditis elegans. J Bacteriol 186, 5087-5092.

Towbin, H., Staehelin, T. \& Gordon, J. (1979). Electrophoretic transfer of proteins from polyacrylamide gels to nitrocellulose sheets: procedure and some applications. Proc Natl Acad Sci U S A 76, 4350-4354.

Wang, R. F. \& Kushner, S. R. (1991). Construction of versatile lowcopy-number vectors for cloning, sequencing and gene expression in Escherichia coli. Gene 100, 195-199.
Wang, X., Preston, J. F., III \& Romeo, T. (2004). The $p g a A B C D$ locus of Escherichia coli promotes the synthesis of a polysaccharide adhesin required for biofilm formation. J Bacteriol 186, 2724-2734.

Wood, P. J. (1980). Specificity in the interaction of direct dyes with polysaccharides. Carbohydr Res 85, 271-287.

Zogaj, X., Nimtz, M., Rohde, M., Bokranz, W. \& Römling, U. (2001). The multicellular morphotypes of Salmonella typhimurium and Escherichia coli produce cellulose as the second component of the extracellular matrix. Mol Microbiol 39, 1452-1463. 